\title{
DER MEXIKANISCHE PRÄSIDIALISMUS UND DIE VERFASSUNG
}

\author{
Von FRIEDRICH WeHNER
}

I.

Der Wechsel im mexikanischen Präsidentenamt Anfang Dezember 1976 erfolgte im Zeichen einer sonst unbekannten politischen und wirtschaftlichen Unruhe. Der bisherige Präsident Luis Echeverría hatte kurz vor seinem Ausscheiden spektakuläre Entscheidungen getroffen durch eine zeitlich und in der Höhe unerwartete Abwertung des Peso und durch eine unvermittelt angeordnete Enteignung einer größeren Zahl von Gütern im Staate Sonora. Devisenabflüsse und Gerüchte über ein Eingreifen des Militärs waren die Folge, ungewohnt in Mexiko seit mindestens den vierziger Jahren. Auffallend für eine demokratische Regierung, wie sie es nach der Verfassung sein soll, ist die autoritäre Art, in der präsidiale Entscheidungen von solcher Tragweite getroffen werden. Weisen die Folgen dieser Ereignisse darauf hin, daß das politische System Mexikos in eine kritische Phase getreten ist? Aus dieser Situation heraus erscheint es angebracht, nach den politischen und verfassungsmäßigen Grundlagen des mexikanischen Regierungssystems zu fragen, die seit rund einem halben Jahrhundert stabilere Verhältnisse geschaffen haben als in der Mehrzahl der lateinamerikanischen Länder. Als Herzstück des Systems hat sich eine bisher unangefochtene überragende Machtposition des Präsidenten herauskristallisiert, die als „Präsidialismus“ gekennzeichnet wird. Wie sich diese Regierungsform in die geltende Verfassung von 1917 mit ihrer liberal-demokratischen Grundlage einfügt, ist $\mathrm{zu}$ untersuchen.

In kontinuierlichem sechsjährigen Wechsel übernehmen seit 1946, als der letzte Generalspräsident Availa Camacho das Amt an Miguel Alemán übergab, nicht mehr Militärs, sondern zivile Persönlichkeiten das Amt. Hier liegt eine wesentliche Grundtatsache für die Konsolidierung des mexikanischen Systems durch die in anderen Ländern Lateinamerikas noch nicht gelungene Ausschaltung des militärischen Caudillismus. Die Voraussetzungen hierfür wurden von Alvaro Obregón, Präsident von 1920-1924, geschaffen und von seinem Nachfolger Plutarco Elías Calles (1924-1928) tatkräftig ausgebaut. Aktionen zur Ausschaltung revolutionärer rivalisierender Generäle bis zu ihrer physischen Liquidierung gingen parallel mit Maßnahmen zur Professionalisierung der Streitkräfte. Obregón, einer der führenden und fähigsten Köpfe aus den Revolutionsjahren seit 1910, faßte die bis dahin oft noch ohne Ausbildung und Disziplin zusammengewürfelten revolutionären Truppen zusammen und reihte Offiziere und Mannschaften in eine reguläre Armee ein, die nicht ohne hartes Durchgreifen gesäubert und reformiert werden konnte.

Der Heeresetat hatte 1917 noch 72 Prozent des Bundesetats betragen, unter Präsident Calles und seinem Kriegsminister Joaquín Amaro wurde die Heeresstärke auf 50000 Mann reduziert, der Etat auf 28 Prozent verringert. Das Interesse der Offiziere wandte sich dem Dienst in einer regulären Armee zu. Die politische Entmachtung der Militärs war bereits imLaufe der zwanziger Jahre so weit gelungen, daß Präsident Calles am 10. September 1928 das Ende der caudillistischen Epoche verkünden konnte. Im weiteren Verlauf unter den Präsidenten Lázaro Cárdenas (1934-1940) und Avila Camacho (1940-1946) wurde die Ausgliederung des 
Militärs aus dem politischen Bereich auch organisatorisch vollendet. Im Rahmen der 1929 von Calles gegründeten Einheitspartei war zunächst das Militär noch als eine der Untergliederungen (Sektoren) berücksichtigt worden; unter Präsident Camacho wurde dieser Sektor 1940 reibungslos aufgelöst. Der Anteil des Militärbudgets hat sich laufend vermindert, 1963 betrug er 6,15 Prozent.

II.

Die heute gültige Verfassung wurde nach nur rund zweimonatigen Debatten in der Stadt Querétaro, der Hauptstadt des gleichnamigen Bundeslandes, am 5. Februar 1917 verkündet. In der verfassunggebenden Versammlung standen sich zwei Gruppen gegenüber, die Vertreter einer bloßen Reform der Verfassung von 1857 und die „Revolutionäre“, denen es um grundsätzliche soziale Reformen ging. Die letzteren setzten sich durch ${ }^{1}$. Unter Beibehalt der liberal-rechtsstaatlichen Konstruktion auf der Basis der Teilung der drei Gewalten Legislative, Exekutive und Justiz wurden umfangreiche Bestimmungen über ein neues Bodenrecht mit Agrarreform (Art. 27), das Monopolverbot mit dem Prinzip der freien Konkurrenz (Art. 28) und ein soziales Arbeitsrecht (Art. 123) eingefügt. Durch die Aufnahme materieller sozialer Forderungen ist die in der Grundkonstruktion an dem westlichen Modell orientierte liberale Verfassung uneinheitlich und zwiespältig geworden. Sie ist nicht mehr die reformierte Ausgabe der Verfassung von 1857, wie es im Titel heißt, sondern die Verfassung von 1917 ist praktisch eine neue Verfassung ${ }^{2}$, in der es zunächst offen bleiben mußte, ob nach den vergeblichen Bemühungen im XIX. Jahrhundert die liberale Grundkonzeption rezipiert werden würde, oder ob sich die sozialreformerischen Elemente durchsetzen würden. Es sollte sich bald zeigen, daß der aus der Revolution ab 1910 gewonnene Schwung stark genug war, um den neuen Ideen Geltung zu verschaffen.

Eine der einschneidenden Veränderungen liegt in dem Art. 27. Der gleichnamige Artikel 27 der Verfassung von 57 stellte entsprechend der liberalen Auffassung lediglich fest, das Eigentum wäre frei und Enteignungen könnten nur aus Gründen des öffentlichen Nutzens und nur gegen Entschädigung erfolgen. Der Art. 27 der neuen Verfassung bietet dagegen einen umfangreichen Katalog von Bestimmungen über das Eigentum an Boden und Gewässern (propiedad de las tierras y aguas) und über die Agrarreform. Das Grundeigentum auf und unter der Erde gebührt (corresponde) originär (originalmente) der Nation. Sie hatte und hat das Recht, solches Eigentum an Personen zu übertragen, wodurch Privateigentum entsteht. Darin liegt die Rückkehr zu vorspanischen Auffassungen und solchen aus der Zeit der Herrschaft der spanischen Krone, in denen die Verfügungsgewalt über den Boden der Öffentlichen Gewalt zustand, soweit nicht ein „justo título“ vorlag. Gem. Art. 27 können dem Privateigentum jederzeit Auflagen (modalidades) erteilt werden.

Über das Eigentum an beweglichen Gütern enthält die neue Verfassung keine ausdrückliche Aussage. Dies Eigentum kann aber auch nur im Zusammenhang mit dem sozialen Gehalt der Verfassung verstanden werden, der individualistische Begriff, nach Belieben mit dem Eigentum verfahren zu können, paßt nicht mehr in das soziale Konzept. Das gilt auch für Art. 28, der das Monopolverbot und die freie

\footnotetext{
1 E. V. Niemeyer jr., Revolution at Querétaro. Austin and London 1974.

2 Jorge Carpizo, La Constitución Mexicana de 1917. México DF (Unam), 1969, S. 147. f. - Alfonso Noriega C, La naturaleza de las garantías individuales en la Constitución de 1917. México DF (Unam), 1967. - S. $74 \mathrm{f}$.
} 
Konkurrenzwirtschaft vorschreibt für Produktion, Industrie, Handel und Dienstleistungen, um die Allgemeinheit vor übertriebenen Preisbildungen zu schützen. Die freie Konkurrenzwirtschaft wird jetzt vom Staate gewährt, sie ist nicht mehr das vorstaatliche individualistische Recht des klassischen Liberalismus.

In Art. 123 werden die Grundlagen eines modernen Arbeitsrechtes statuiert, wofür es in der Verfassung von 1857 kein Vorbild gibt. Die Bestimmungen dieses Artikels sind inzwischen durch Spezialgesetze ausgebaut worden, insbesondere durch das Bundesarbeitsgesetz vom 1. Mai 19703.

Durch Aufnahme dieser sozialen Elemente wurde die neue Verfassung zum „Evangelium der Revolution“, zur „juristischen Konstituierung der sozialen Wünsche des Volkes"4. Die neue Verfassung war keine Imitation des westlich-industriellen Modells mehr, sie hat einen authentisch mexikanischen Charakter ${ }^{5}$. Die Revolution von 1910 war zunächst von Persönlichkeiten liberaler Prägung getragen worden, an ihrer Spitze Francisco I. Madero. Ihnen kam es darauf an, die liberale Grundkonstruktion der Verfassung von 1857 mit dem Prinzip der Gewaltenteilung zu erhalten, sie nach der langährigen Diktaturperiode des Porfirio Díaz zu stabilisieren und mit einigen Reformzusätzen zu versehen. Der letzte bedeutende Vertreter dieser Richtung war Venustiano Carranza, Träger der „konstitutionellen“ Bewegung und erster Präsident von 1917-1920. Mit seinem Tode 1920 fand diese Richtung ihr Ende. Von da ab bestimmten die Angehörigen der „Revolutionären“ oder "Politischen Familie" die Geschicke, die sich als Kern der Revolutionäre gebildet hatte. Durch diese Revolution und die verfassungsmäßige Festlegung auf moderne soziale Ziele hat Mexiko - im Gegensatz zur Mehrzahl der lateinamerikanischen Staaten - schon früh den Abschied vom XIX. Jahrhundert und den Úbergang zum modernen Sozialstaat des XX. Jahrhunderts vollzogen. Die in Querétaro beschlossene Verfassung war eine echte politische Entscheidung. Sie hat die Basis für eine konstante Entwicklung und Stabilisierung Mexikos gelegt und ist heute, sechzig Jahre später, immer noch die bleibende Grundlage des Staates. Aus der Verfassung in Verbindung mit historischen Gegebenheiten ist die heutige Verfassungswirklichkeit gewachsen.

III.

Neben der Verdrängung des militärischen Caudillismus wird die neue Verfassungswirklichkeit entscheidend durch die Bildung eines von oben her dirigierten Parteiwesens bestimmt. Es setzt sich aus einer „offiziellen“, von der Regierung in ihrem Sinne gelenkten Partei sowie aus drei oppositionellen Parteien von geringerer politischer Bedeutung zusammen.

Die offizielle Partei, die heutige „Partido Revolucionario Institucional“, abgekürzt $\mathrm{PRI}^{6}$, kam auf eine unorthodoxe Weise zustande, nicht nach dem üblichen liberaldemokratischen Hergang durch bürgerliche Initiative von unten, sondern durch eine Verfügung des damals gerade aus dem Präsidentenamt abtretenden Plutarco Elías Calles, der 1929 die Partei unter dem Namen „Partido Nacional Revolucionario“ (PNR) gründete, um das Parteiwesen auf den Weg der Institutionalisie-

3 Peter Schulenburg, Arbeitsrecht in Mexiko. Schriftenreihe des Instituts für Iberoamerika-Kunde, Hamburg, Heft 21.

4 Carpizo, a. a. O., S. $149 \mathrm{f}$.

5 Antonio Carrillo Flores in: El Pensamiento Contemporaneo en México. - México 1974. - S. 182.

6 Robert K. Furtak, Revolutionspartei und politische Stabilität in Mexiko. Schriftenreihe des Instituts für Iberoamerika-Kunde, Hamburg, Heft 12. - Frank Brandenburg, The Making of Modern Mexico. Englewood Cliffs (NJ), 1964. - Pablo González Casanova, La Democracia en México, México DF 1974, 6. Aufl. - Frank Tannenbaum, Mexico - The Struggle for Peace and Bread. New York 1964. 
rung zu führen; er selbst übernahm den Vorsitz. Vorher hatte es parteiähnliche Gruppierungen gegeben, ihrem Wesen nach waren sie personalistische Anhängerschaften um einen Caudillo ohne festes Programm. Sie wurden entsprechend bezeichnet als Porfiristas, Maderistas, Zapatistas, Villistas, Obregonistas, Callistas, Carrancistas - eine Reihenfolge, aus der sich die personalistische Struktur der Jahrzehnte vor und nach 1910 ablesen läßt. Calles stand 1929 unter dem Zwang, die „Politische Familie“, den elitären Kern der Revolutionäre, dem der Zerfall drohte, als aktive Kraft und inneren Stabilisierungsfaktor wieder zusammenzufassen. Die Partei, die die "mexikanischste aller Parteien“ genannt worden ist, entwickelte sich rasch zu ihrer zentralen Bedeutung; im Jahre 1946 erhielt sie den heute noch gültigen Namen „Partido Revolucionario Institucional“ - PRI.

Die Zielsetzung der PRI ist gerichtet auf Vollendung der in der Verfassung verankerten Reformvorhaben; ihre wichtigste Aufgabe ist die Durchführung friedlicher Wahlen, wobei sie sich besonders als Instrument der Regierung bewährt hat. Führung und Organisation der Partei sind zwar nicht mit der Staatsführung gekoppelt, die PRI ist formell und organisatorisch selbständig, eine Personalunion zwischen Bundespräsident und Parteivorsitzendem besteht nicht. Dennoch darf man davon ausgehen, daß alle wichtigen personellen und sachlichen Entscheidungen dem Placet des Präsidenten unterliegen.

Die Mitgliedschaft wird erworben in erster Linie durch korporative Eingliederung der großen Dachorganisationen, die alle Arbeitnehmerorganisationen umfassen, die in der PRI in drei „Sektoren“ gegliedert sind:

die "Confederación Nacional de Campesinos" - CNC - erfaßt alle zentralen und regionalen Verbände der Landarbeiter

die "Confederación de los Trabajadores Mexicanos - CTM - alle gewerblichen Arbeiter

der Sector Popular mit der "Confederación Nacional de Organizaciones Populares - CNOP - die Beamten, Angestellten und den mittelständischen Bereich.

Die PRI sieht sich als Vertreterin aller wirtschaftlich und gesellschaftlich unterprivilegierten Teile der Bevölkerung. Durch diese Partei wird die Masse der Wählerschaft an die offizielle Regierungslinie gebunden und von ihr kontrolliert. Das Entstehen autonomer Massenbewegungen und eines autonomen Verbandswesens, das die Staatlichkeit in Frage stellen könnte, wird damit anders als in parlamentarisch regierten Staaten Westeuropas ausgeschaltet ${ }^{7}$. In den genannten drei Sektoren ist kein Platz für die Berufsvereinigungen der gewerblichen Unternehmerschaft und des Großgrundbesitzes, da ihre Interessen als den revolutionären Zielen der sozialen Gerechtigkeit zuwiderlaufend angesehen werden. Für die Angehörigen dieser Kreise besteht seit 1940 die Möglichkeit des Erwerbs der Einzelmitgliedschaft. Ihr politischer Einfluß ergibt sich aus ihrer wirtschaftlichen Macht in direktem Zugang zu Präsident und Exekutive.

Die wichtigste Funktion der PRI im Regierungssinne ist die Durchführung und Steuerung aller öffentlichen Wahlen. Alle Wahlämter sind in der Hand der PRI, in deren Rahmen die Kandidaten aller Stufen parteiintern ausgewählt und präsentiert werden. Die ermittelten Kandidaten werden dann „spontan“ gewählt, die in der Partei disziplinierte Masse der Wählerschaft garantiert den Erfolg.

7 Zur Situation in der Bundesrepublik Deutschland - siehe Ernst Forsthoff, der Staat der Industrie-Gesellschaft. München 1971. 
Gegenüber der PRI treten die übrigen drei Parteien in der Bedeutung zurück ${ }^{8}$. Sie müssen zugelassen und registriert sein. Sie sind aus Bürgerinitiativen entstanden, ihr Wählerpotential ist ebenso wie ihr politischer Einfluß durch die erdrückende Macht der PRI begrenzt. Das Mehrheitswahlrecht vermindert ihre Chancen zusätzlich.

Die bedeutendste dieser zugelassenen Parteien ist die „Partido Acción Nacional“ PAN, die 1939 gegen den zu betonten Linkskurs des Präsidenten Cárdenas entstand. Sie sammelt vornehmlich Personen aus Kreisen der Unternehmer und der freien Berufe mit konservativer Einstellung und rekrutiert sich vorwiegend aus den großen Städten. Sie ist die einzige Oppositionspartei von einiger Bedeutung, da sie über einen gewissen internen politischen Einfluß der ihr angehörenden führenden Wirtschaftskreise verfügt. Seit 1952 beteiligt sie sich auch an den Präsidentenwahlen und hat im Abgeordnetenhause Fuß gefaßt. Ihre Mitgliederzahl ist von ca. 100000 (1954) auf rd. 750000 (1967) gestiegen.

Links von der PRI siedelte sich seit 1960 die „Partido Popular Socialista“ — PPS an, deren Mitglieder sich aus unzufriedenen marxistischen und anderen Linkskräften rekrutieren. Seit 1955 bekennt sie sich zum wissenschaftlichen Sozialismus, lehnt aber - typisch für den mexikanischen Pragmatismus - ein doktrinäres Verhalten für einen sozialistischen Aufbau des Landes ab.

Die dritte seit 1957 zugelassene Partei ist die „Partido Auténtico de la Revolución Mexicana“ - PARM; sie setzt sich für Erneuerung des revolutionären Elans ein.

Die drei Parteien können nur eine begrenzte Anzahl von Abgeordneten im Parlament erreichen, und zwar einmal aus direkten Wahlergebnissen (diputados de mayoría) und zum anderen durch Zuschlagung bestimmter zusätzlicher Mandate (diputados de partido) insgesamt mit einer Höchstzahl von je zwanzig, nach einer Wahlrechtsreform von 1963. Die Sitzverteilung im Abgeordnetenhaus sieht wie folgt aus ${ }^{9}$ :

\begin{tabular}{|c|c|c|c|c|c|}
\hline & PRI & PAN & PPS & PARM & Total \\
\hline \multirow{3}{*}{1964} & & 2 & 1 & 0 & \\
\hline & & $\left.+18^{*}\right)$ & $+9: 5$ & $+5 \%)$ & \\
\hline & 175 & $=20$ & $=10$ & $=5$ & 210 \\
\hline \multirow{3}{*}{1967} & & 1 & 0 & 1 & \\
\hline & & $+19 *)$ & $+10 *)$ & $+5 \%$ & \\
\hline & 172 & $=20$ & $=10$ & $=6$ & 208 \\
\hline \multirow{3}{*}{1977} & & 0 & 0 & 1 & \\
\hline & & $+20 *)$ & $+12 *)$ & $+9 *)$ & \\
\hline & 195 & $=20$ & $=12$ & $=10$ & 237 \\
\hline
\end{tabular}

*) = die offiziell zugeschlagenen Mandate (diputados de partido)

Mit diesen schwachen Anteilen im Abgeordnetenhause können diese Parteien keine wirkungsvolle Opposition betreiben, eine direkte Beteiligung an der Regierung bleibt ihnen versagt.

8 Furtak, a. a. O., 61 ff., 104 ff. - Carpizo, a. a. O., S. 273, Anm. 489. - Cosío Villegas, Daniel, El Sistema político mexicano. México 1974, S. $68 \mathrm{ff}$

9 Furtak a. a. O., S. 65. - Rafael Preciado Hernández, Presupuesto de la Reforma democrática en México. In: Los Problemas Nacionales, México 1971, S. 137. 
IV.

Die heutige umfassende Machtposition des Präsidenten fußt zunächst auf seinen verfassungsmäßigen Rechten und ferner auf Elementen, die sich im Zuge der Entwicklung der neuen Verfassungswirklichkeit seit 1917 herauskristallisiert haben. Schon nach dem Text der Verfassung verleiht die von den USA übernommene Form der Präsidialdemokratie dem Präsidenten eine größere Machtfülle, als sie einem Präsidenten einer parlamentarischen Demokratie zusteht. Die Präsidialdemokratie ist eine monokratische Form der Demokratie ${ }^{10}$, in der der Präsident zugleich Staatsrepräsentant und Ministerpräsident ist. Er ernennt und entläßt seine Staatssekretäre (Minister) aus eigenem Ermessen (Art. 89 [II ff.]). Die Verfassunggeber von Querétaro haben diese schon in früheren Verfassungen enthaltene Form bewußt beibehalten und sogar noch gestärkt, als Reflex auf die von den Verfassunggebern von 1857 bevorzugte Minderung seiner Position durch indirekte Wahl, um damals die Legislative zu stärken, die in den ersten dreißig Jahren seit 1824 infolge eines Übermaßes an Diktaturen an Ansehen verloren hatte. 1917 war die Ausgangslage umgekehrt: Man wollte die Position des Präsidenten durch Direktwahl als eines unmittelbaren Mandates des souveränen Volkes herausheben (Art. 81), auch im Hinblick auf Erfahrungen mit ineffektiven parlamentarischen Debatten.

Durch das Instrument der offiziellen Partei PRI ist der Exekutive in der Person des Präsidenten heute eine zusätzliche Machtfülle zugewachsen, die sich mit der liberalen Grundkonstruktion der Verfassung und deren Prinzip der Teilung der Gewalten nicht mehr vereinbaren läßt. Der Artikel 49 der Verfassung besagt eindeutig, daß sich die Höchste Gewalt für ihre Durchführung in die Legislative, die Exekutive und die Justiz aufteilt. Weder dürfen zwei oder mehr dieser Funktionen in der Hand einer Person oder Korporation vereinigt werden, noch darf die Legislative durch eine Person ausgeübt werden. Eine Ausnahmeregelung ist gem. Art. 29 für den Fall einer Invasion oder einer schweren Störung des öffentlichen Friedens nur insoweit vorgesehen, als dem Präsidenten zeitlich limitiert gestattet wird, Grundrechte außer Kraft zu setzen. Die Häufigkeit der Anwendung dieser Vorschrift, die gleichlautend auch in der Verfassung von 1857 enthalten war, ist immer strittig gewesen, zumal es angesichts der Notwendigkeit, das Land diktatorisch regieren $\mathrm{zu}$ müssen, zur gewohnheitsrechtlichen Praxis geworden war, den Präsidenten mit außerordentlichen Vollmachten auszustatten, die ihm sogar gesetzgeberische Tätigkeit ermöglichte und ihn der Kontrolle des Parlamentes entzog - ein verfassungsrechtlicher Mißbrauch ${ }^{11}$. Sogar nach Verkündung der Verfassung von 1917 ließ sich der erste Präsident Venustiano Carranza schon im Jahre 1917 eine außerordentliche haushaltrechtliche Ermächtigung erteilen.

In der weiteren Entwicklung wurde, infolge ständiger Stärkung der Position des Präsidenten, nicht zuletzt durch die Verbundenheit mit der PRI, das Prinzip der Gewaltenteilung endgültig außer Kraft gesetzt. Die Mitglieder des Senates und die überwiegende Zahl der Abgeordneten sind Parteimitglieder. Die verfassungsmäßige Kontrolle der Exekutive durch den Kongreß entfällt ebenso wie auch die ausschließliche Gesetzgebung durch den Kongreß. Der eigentliche Gesetzgeber ist heute der Präsident, dem Kongreß verbleibt es, die Vorlagen des Präsidenten formell zu legalisieren; als legative Gewalt ist der Kongreß entmachtet.

10 Carl Joachim Friedrich, Demokratie als Herrschafts- und Lebensform, Heidelberg 1966, S. 30. 
Neben Exekutive und Legislative ist in der Verfassung als dritte Gewalt die Justiz vorgesehen, der aber in dem mexikanischen System schon verfassungsmäßig nicht die Kraft zugemessen worden ist, um eine umfassende kontrollierende und regulierende Rolle spielen zu können. Die Mexikaner stehen einer Ausübung politischen Einflusses und einer Unterordnung der autoritären Gewalt unter die Justiz grundsätzlich skeptisch gegenüber. Mexiko hat das amerikanische System übernommen, wonach alle aus Fragen der Verfassungsmäßigkeit entstehenden Zweifel der Entscheidung der ordentlichen Gerichtsbarkeit unterliegen. Eine spezielle Verfassungsgerichtsbarkeit, wie in der Bundesrepublik Deutschland, gibt es nicht. Mexiko hat aber seit der Verfassung von 1847 in dem AMPARO-Verfahren ein Rechtsinstitut geschaffen, das dem Einzelnen prozessuale Möglichkeiten gibt, sich gegen Verfassungsverstöße der Exekutive oder der Legislative zu wehren, um die Innehaltung der in der Verfassung gewährten "Garantías Individuales“ (Art. 1-29) sicherzustellen. Eine in einem solchen Verfahren festgestellte Verfassungswidrigkeit hat keine allgemeine Wirkung "erga omnes“, es bleibt den Organen überlassen, hieraus Schlüsse zu ziehen oder es zu unterlassen. Auch tritt dies Verfahren nur in Kraft, wenn der sich geschädigt Fühlende Klage erhebt. In den letzten Jahrzehnten hat sich die Diskussion zu diesem Thema verstärkt; die europäische Methode der Einrichtung von Verfassungsgerichten zum unmittelbaren Schutz der Verfassung hat sich nicht durchsetzen können ${ }^{12}$.

Es entspricht allen mexikanischen Rechtstraditionen, daß die Macht des Präsidenten nicht unbegrenzt, nicht absolut ist. Trotz der weitgehenden Unabhängigkeit, seine Intentionen nach eigenem Ermessen zu verwirklichen, wäre es unzutreffend, den Präsidenten als Diktator im Sinne der absoluten ungebundenen Diktatoren des XIX. Jahrhunderts zu bezeichnen. Das Pathos der Revolution lag in der Forderung "Sufragio efectivo - no reelección“, Direktwahl des Präsidenten, aber ohne Wiederwahl (Art. 83, 85). Diese Grundforderung ist zum Dogma geworden. Eine Hervorhebung der Position des Präsidenten ist ebenso Grundlage des Systems wie die zeitliche Begrenzung seines Wirkens auf sechs Jahre. Eine Wiederholung von unkontrollierten Diktaturen und der anarchischen Zustände unter rasch sich ablösen den Caudillos in der ersten Hälfte des XIX. Jahrhunderts, zuletzt dann in der 34 Jahre dauernden Diktatur des Porfirio Díaz von 1876-1910 mußte vermieden werden. Seit 1934 hat sich ein regelmäßiger Wechsel im Amt des Präsidenten vollzogen, der dem Amtsträger für sechs Jahre die volle Macht überläßt, ihm aber die Festsetzung in der Macht versperrt.

Eine unübersehbare sachliche Begrenzung liegt in der Verpflichtung auf die revolutionären Reformziele. Gegen sie darf ebenso wenig verstoßen werden wie gegen das stark entwickelte Nationalempfinden der Mexikaner, das in der Verfassung in zahlreichen Bestimmungen zum Ausdruck kommt. Aus den in den Art. 27 und 28 vorgezeichneten Linien ist eine "economía mixta“ erwachsen, in der der Staat wichtige Grundstoffindustrien und infrastrukturell wichtige Bereiche durch staatseigene Betriebe lenkt und den Bereich der freien Konkurrenzwirtschaft absteckt.

11 Felipe Tena Ramirez, Derecho Constitucional Mexicano. México 1967, 8. Aufl., S. $213 \mathrm{ff}$.

12 Tena Ramirez, a. a. O., S. 451 ff. Héctor Fix-Zamudio, Verfassungskontrolle in Lateinamerika. In: Jahrbuch des Offentlichen Rechts der Gegenwart, Bd. 25, 1976, S. 649 ff. - Ds., Veinticinco Años de Evolución de la Justicia Constitucional 1940-1965. México DF, 1968. Niceto Alcalá Zamora y Castillo, Judicial Protection of the Individual against the Executive in Mexico. In: Gerichtsschutz gegen die Executive. Carl Heimann, Köln 1970, S. $771 \mathrm{f}$. - Hans-Rudolf Horn, Das Amparo-Verfahren in Mexiko: In VRU Heft 2/1968, S. $162 \mathrm{ff}$. - Hans Christoph von Rohr, Der argentinische AmparoProzeß. Bonn 1969, S. 26 ff., 97. - Carpizo, a. a. O., S. $320 \mathrm{ff}$. 
Industrieförderung wird verbunden mit Vorschriften über den Höchstanteil ausländischer Investitionen ${ }^{13}$. Die heutige Wirtschaftsform der „economía mixta“ wird häufig unzutreffend als Mischung von Kapitalismus und Sozialismus bezeichnet, was ihr eine ideologische Charakterisierung verleihen würde, die dem pragmatischen Stil der Regierungsführung der Präsidenten nicht entspricht. Auch der jetzige Präsident José López Portillo hat bekundet, das System wäre „ni socialismo - ni capitalismo"14. Auf die Gefahren einer Verquickung von Regierung und Geschäft und einer oligarchischen Verkrustung sei hier nur am Rande hingewiesen ${ }^{15}$.

Gegen die ermessensfreie und ihrem Wesen nach pragmatische Regierungsführung der Präsidenten erhebt sich kein prinzipieller Widerspruch im Volke, wohl aber Unzufriedenheiten infolge bisher unzureichender Erfüllung der sozialen Forderungen, unter denen das Agrarproblem an erster Stelle steht. Das mexikanische Volk empfindet offenbar die heutige Form autoritären Regierens als legitim und akzeptiert sie mindestens stillschweigend als „consenso real“. Wer die revolutionären Ziele verfolgt, handelt legitim ${ }^{\mathbf{1 6}}$. Geschriebenes Recht begründet die Legalität, nicht unbedingt auch die Legitimität; geschriebenes Verfassungsrecht schließt ungeschriebenes, aus der Geschichte gewordenes Recht nicht aus ${ }^{17}$.

Die Begründung der Legitimität folgert aus der mexikanischen Geschichte. Monokratische Regierungen waren die Normalform der politischen Führung. Durch die Geschichte zieht sich eine Linie von der auroritär-hierarchischen Herrschaft im Aztekenreich, vom "tlatoani“, dem "Ersten Sprecher" der Azteken, über die monarchische Staatsführung der spanischen Krone bis zum heutigen Präsidenten, dessen Position der einer auf sechs Jahre begrenzten Monarchie ähnelt, gewissermaßen sogar erblich, da der Nachfolger aus dem inneren Kreis der "Politischen Familie“ bestimmt wird ${ }^{18}$. Alle Bemühungen um die Einführung eines Staates der liberalen Demokratie seit 1824 haben diese historische Gesetzmäßigkeit mißachtet. Das Prinzip der Gewaltenteilung ist zu konträr zu den mexikanischen Vorstellungen von der Einheit der autoritären Regierung. Die heutige Ausformung des Präsidialamtes hat eine mehr als hundert Jahre dauernde Epoche des Ubbergangs, des vergeblichen Bemühens um Integrierung des fremden Verfassungsmodells beendet, eine Epoche, die man als Verfassungsinterregnum bezeichnen kann.

Auch in Zeiten dieses Interregnums war der Präsident immer das Zentrum der Macht geblieben, das dominierende Organ des Staates ${ }^{19}$. Immer wurde und wird der Präsident direkt und persönlich angesprochen als das Emblem der Macht. Auch für den letzten Indio ist er die institutionelle Figur des „El Señor Presidente“. Der Präsident ist die oberste Schiedsinstanz, die "supremo arbitraje“. Die Präsidentschaft hat institutionellen Charakter, sie gilt im Grundsatz nicht den wechselnden Trägern des Amtes. Die Verfassungswirklichkeit des modernen Mexiko ist aus der Geschichte heraus gewachsen als "Präsidialismus“, „precidialismo mexicano“ oder

13 Henning von Wedel, Neue Mexikanische Wirtschaftsgesetze, In VRU Heft 1/1974, S. 59 ff.

14 Excelsior, 24. 6. 1975 .

15 Hierzu s. Christian Graf von Krockow, Mexiko. München 1974, S. 63 ff

16 Victor Flores Olea in: El Perfil de México en 1980: Poder, Legitimidad y Política en México. México 1972. - S. 482.

17 Carl Joachim Friedrich, a. a. O., S. 30. S. a. Konrad Hesse, Grundzüge des Verfassungsrechtes der Bundesrepublik Deutschland. - 9. Aufl. 1976.

18 Daniel Cosío Villegas, a. a. O., S. $27 \mathrm{ff}$. Victor Flores Olea in „Los Problemas Nacionales“, México 1971 , S. 119.

19 Carpizo, a. a. O., S. 352, 361. 
auch „régimen precidialisto“20, als die typisch mexikanische Prägung des spanischamerikanischen ,authoritism“ 21 .

V.

Nach der Verfassung ist Mexiko eine demokratische Republik, was nach allem Gesagten nicht im Sinne einer liberalen Demokratie nach dem Vorbild westlicher Industriestaaten verstanden werden kann. Eine Annahme, der augenblickliche $\mathrm{Zu}-$ stand sei nur eine Etappe auf dem Wege zu einer „echten“ Demokratie, erscheint abwegig, dem steht immer die "mexicanidad“ und die geringe Aufnahmebereitschaft für fremde Ideologien im Wege; man ist eher besorgt, die „mexicanidad“ würde von fremden Einflüssen bedroht ${ }^{22}$. Auch im System des "precidialismo“ wird dem Volke eine immer breitere Mitwirkung eingeräumt werden müssen, was aber nicht im Sinne einer Demokratie nach westlichen Vorstellungen verstanden sein kann. Aus der politischen Entwicklung Mexikos folgert die Frage, ob überhaunt das demokratische Modell des Westens für immer und für alle Zeiten und Völker das „richtige“ Modell ist ${ }^{23}$, oder ob nicht auch dies Modell, aus bürgerlichliberalen Initiativen entstanden, nur da gedeihen kann, wo es seine adäquaten historischen und ideologischen Voraussetzungen vorfindet, so daß es in der zeitlichen und sachlichen Anwendbarkeit begrenzt ist. Die mexikanische Verfassungsgeschichte ist ein Beispiel für die Notwendigkeit der Übereinstimmung der Verfassung mit den historischen und ideologischen Gegebenheiten.

In der heutigen Verfassungswirklichkeit aus einer Kombination von legalen Teilen der Verfassung mit einer Eigenentwicklung aus legitimen historischen Reminiszenzen ist die Grundkonstruktion der Dreiteilung der Gewalten im liberalen Sinne nicht rezipiert worden. Wenn trotzdem die betreffenden Formulierungen stehen blieben und eine Divergenz zwischen Teilen des Textes der Verfassung und der Wirklichkeit erscheint, und wenn keine Anstalten unternommen werden, diese Divergenz zu tilgen, so entspricht dieser Zustand spanischer Rechtstradition. Die Kluft zwischen der Form des königlichen Gesetzes und seiner oft verweigerten Anwendung mit dem Vermerk „se acata, pero no se cumple“, dem Gesetz wird die nötige Ehrerbietung bezeugt, aber es läßt sich leider nicht anwenden, die Kluft zwischen „derecho y hecho“, war ein spezifisches Merkmal der spanischen Kolonialepoche.

20 Ds., S. 347.

21 Dargestellt am Beispiel Chile: Francisco José Moreno, Legitimacy and Stability in Latin America. New York 1969

22 Andrés Serra Rojas, La Función Constitucional del Presidente de la República. - In: El Pensamiento Jurídico de México. México 1962 (Porrua), S. 221.

23 S. a. Manfred Mols in VRU Heft $1 / 1975$, S. 5 ff. 


\title{
Constitutionalism and Presidency in Mexico
}

\author{
BY F. WEHNER
}

The Constitution of Querétaro, dated 5th of February of 1917, was intended to be a reformed edition of the Constitution of 1857. In the constitutional reality as it has been developped since 1917, the Constitution is no longer an imitation of the model of USA, but is to be understood as an originally Mexican new Constitution. It contains elements of a socialrevolutionary character, such as a definition of the property on the soil which corresponds Aztec and Spanish traditions with the intention to spoil the privileges of the latifundists; besides new modern basic normes of a social right are stated.

During the years of the twentieths the two Presidents Alvaro Obregón (19201924) and Plutarco Elías Calles (1924-1928) succeeded in fighting down the traditional military Caudillism. Another predominating fact was the founding of an official Party, the "Partido Revolucionario Institucional" - PRI, which stabilized the presidential government acting as an instrument to manage the elections and to dominate the masses of the people.

On this basis the position of the President has reached a highly authoritative character confirming thus the tradition and constitutional reality throughout the Mexican history. Nevertheless the position of the President cannot be compared with absolutism. He is bound to observe the tasks of social progress according to the Constitution and the period of his power is limited to six years without any come-back. This was a dogma of the Revolution of 1910. People acknowledge the presidential power as legitime. Though it is contrary to elements of the written Constitution which still prescribes the division of the legislative, executive and judicial powers. This liberal dogma is no longer in action.

\section{The Judicial System in Mexico}

\section{By Hector Fix-Zamudio}

An analysis of the constitutional origins of the Mexican Judicial System and its evolution during the last 25 years does not show a dynamic evolution, neither on the federal nor on the state level. As far as the constitutional reforms of 1951 and 1968 are concerned, these reforms only intended to relieve the courts - especially the Supreme Court of Justice- from their enormous back-lag in amparo-matters. This has been achieved with regard to the Supreme Court, but only partially with regard to other federal courts. The Supreme Court of Justice has done important work in the field of interpreting the country's judicial system, but nevertheless his role as a final authority in interpreting the constitution has not reached the importance of the U.S. Supreme Court or the courts of some European countries. There are two main reasons for this. The first one is, that even after the reforms mentioned above, the Supreme Court, due to his limited competence for selecting the cases to be judged, is still overburdened in amparo-matters. Secondly, in Mexico, one has always preferred to adapt the text of the constitution to the very dynamicly changing social conditions. This seemed to be the more simple way, but 